\title{
WEAK AND UNBOUNDED ORDER CONVERGENCE IN BANACH LATTICES
}

\author{
A. W. WICKSTEAD
}

(Received 1 October 1976)

Communicated by E. Strzelecki

\begin{abstract}
A net $\left(x_{\gamma}\right)$ in a vector lattice is unbounded order convergent (uo-convergent) to 0 if $u \wedge \mid x_{\gamma}$ : order converges to 0 for all $u \geqq 0$. We consider, in a Banach lattice, the relationship between weak and uo-convergence. We characterise those Banach lattices in which weak convergence implies uo-convergence and those in which uo-convergence of a bounded net implies weak convergence. Finaliy we combine the results to characterise those Banach lattices in which weak and $u o$-convergence coincide for bounded nets.
\end{abstract}

\section{Introduction}

The relationship between norm and various forms of order theoretic convergence has been studied quite extensively (see Schaefer (1974), Theorem II.5.10 and Wirth (1975)). In this note we look at the relationship between weak convergence and unbounded order convergence (see below for definition) in Banach lattices. The study is motivated by the spaces $c_{0}$ and $l_{p}$ $(1<p<x)$. In these spaces the two notions of convergence coincide for norm bounded nets.

In $\$ 2$ we ask when weak convergence implies unbounded order convergence. This is equivalent to the linear span of the minimal ideals in the Banach lattice being order dense. It does not suffice in the proof of this result to consider only sequences, as we show by an example, however it would if we assumed that the Banach lattice had an order continous norm.

The final section asks when every norm bounded unbounded order convergent net is weakly convergent (if all unbounded order convergent nets are weakly convergent then the Banach lattice must be finite dimensional). This turns out to be equivalent to the norms in both the Banach lattice and its dual being order continuous. Again, we show that it does not suffice to consider only sequences. The two main results are then combined to 
characterise those Banach lattices for which weak and unbounded order convergence coincide for norm bounded nets.

We adopt Schaefer (1974) as our basic text on Banach lattices and use the terminology therein wherever possible.

\section{When does weak convergence imply uo-convergence?}

Let $E$ be a vector lattice. A net $\left(x_{\gamma}\right)$ in $E$ is said to be order convergent to 0 (or to $o$-converge to 0 ) if there is a net $\left(y_{\delta}\right)$ in $E_{+}$decreasing to 0 such that for each $\delta$ there is $\gamma_{0}$ with $\left|x_{\gamma}\right| \leqq y_{\delta}$ whenever $\gamma \geqq \gamma_{0}$. The net $\left(x_{\gamma}\right)$ is unbounded order convergent to 0 (or uo-converges to 0 ) if $u \wedge\left|x_{\gamma}\right| o$-converges to 0 for all $u \in E_{+}$.

Unbounded order convergence has been used in DeMarr (1964). He gives there some examples of its significance in concrete vector lattices.

THEOREM 1. In any Banach lattice $E$ the following are equivalent:

(i) Every net in $E$ which converges weakly to 0 must uo-converge to 0.

(ii) Every order bounded net in $E$ which converges weakly to 0 must o-converge to 0 .

(iii) The linear span of the minimal ideals in $E$ is order dense in $E$.

Proof. Clearly (i) implies (ii).

Suppose (iii) is false, then there is $x \in E_{+}$such that $x \neq 0$ and $E_{x}$, the ideal generated by $x$ in $E$, has no minimal ideals. $E_{x}$ normed by the gauge of $[-x, x]$ is an $A M$-space with unit so is isomorphic to some space $C(K)$ (Schaefer (1974), Corollary 1 of Theorem II.7.4). Ex having no minimal ideals is equivalent to $K$ having no isolated points. We shall prove that (ii) fails for such a $C(K)$ and hence for $E_{x}$. Now if we have a net $\left(x_{\gamma}\right)$ in $E_{x}$ which is order bounded and converges $\sigma\left(E_{x}, E_{x}^{\prime}\right)$ to 0 then $\left(x_{\gamma}\right)$ converges $\sigma\left(E, E^{\prime}\right)$ to 0 as the natural embedding of $E_{x}$ into $E$ is continuous (Schaefer (1974), Corollary of Proposition II.7.2) and hence weakly continous (Köthe (1969), \$20.4.5). If we choose $\left(x_{r}\right)$ so that it does not $o$-converge to 0 in $E_{x}$ then it will not do so in $E$, so that (ii) fails also for $E$.

Suppose $K$ has no isolated points, and let $v \in C(K)$ with $\delta e_{K} \leqq v<e_{K}$ for some $\delta>0$, where $e_{K}$ is the constantly one function on $K$. I claim that given any basic weak neighbourhood of $0, B=\left\{f \in C(K):\left|\mu_{j}(f)\right|<1\right.$, $\left.\mu_{j} \in C(K)^{\prime}, j=1,2, \cdots, n\right\}, B \cap\left[-e_{K}, e_{K}\right] \not \subset[-v, v]$. Let $\left(k_{i}\right)$ be countably infinitely many distinct points of $K$ with $0<v\left(k_{i}\right)<1$.

Let us identify each $\mu_{i}$ with a regular Borel measure on $K$. I claim that the assumption that $B \cap\left[-e_{K}, e_{K}\right] \subset[-v, v]$ implies that for each $i$ there is $j$ with $\left|\mu_{j}\left(\left\{k_{i}\right\}\right)\right| \geqq v\left(k_{i}\right)^{-1}$. It would follow that 


$$
\sum_{j=1}^{n}\left\|\mu_{j}\right\| \geqq \sum_{j=1}^{n} \sum_{i=1}^{m}\left|\mu_{j}\left(\left\{k_{i}\right\}\right)\right| \geqq \sum_{i=1}^{m} v\left(k_{i}\right)^{-1} \geqq m
$$

for all integers $m$, which is impossible.

Fix $i$ and suppose that $\left|\mu_{i}\left(\left\{k_{i}\right\}\right)\right|<v\left(k_{i}\right)^{-1}$ for $j=1,2, \cdots, n$. Choose $\varepsilon>0$ such that $v(k)+\varepsilon<1$ and use regularity of $\mu$ to find an open subset $P$ of $K$ with $k_{i} \in P$ and $\left|\mu_{j}^{+}(P)\right|+\left|\mu_{j}^{-}(P)\right| \leqq\left(v\left(k_{i}\right)+\varepsilon\right)^{-1}$ for $j=1,2, \cdots, n$. Now construct $g \in C(K)$ with $g\left(k_{i}\right)=v\left(k_{i}\right)+\varepsilon, 0 \leqq g \leqq e_{K}$ and $g$ vanishing on $K \backslash P$. Now $g \in B \cap\left[-e_{K}, e_{K}\right]$ but $g \notin[-v, v]$, contradicting the assumption that $B \cap\left[-e_{K}, e_{K}\right] \subset[-v, v]$.

Let $\mathscr{B}$ be the family of all basic weak neighbourhoods of 0 . Order the set $\left\{(B, v): B \in \mathscr{B}, \delta e_{K} \leqq v<e_{K}\right.$ for some $\left.\delta>0\right\}$ by defining $(B, v) \geqq\left(B^{\prime}, v^{\prime}\right)$ to mean $B \subset B^{\prime}$ and $v \geqq v^{\prime}$. By the arguments of the last three paragraphs, for each $(B, v)$ there is $x=x(B, v) \in\left[-e_{K}, e_{K}\right]$ with $x \in B, x \not v$. The net $(x(B, v))$ converges weakly to 0 , but does not $o$-converge to 0 . For suppose ( $\left.w_{\delta}\right)$ decreased to 0 and for each $\delta$ there was $\left(B_{0}, v_{0}\right)$ with $|x(B, v)| \leqq w_{\delta}$ for $(B, v) \geqq\left(B_{0}, v_{0}\right)$. If $w_{\delta}<e_{K}$ we may choose $v$ such that $e_{K}>v>v_{0}, w_{\delta}$, and then $\left(B_{0}, v\right) \geqq\left(B_{0}, v_{0}\right)$ yet $x\left(B_{0}, v\right) \geqq v$, so $x\left(B_{0}, v\right)$ proof that (ii) implies (iii).

Finally we prove that (iii) implies (i). Suppose that (iii) holds and that $\left(x_{\delta}\right)$ is a net in $E$ which converges weakly to 0 . If $J$ is a minimal ideal in $E$, we let $P_{J}$ denote the band projection onto $J$. Fix $u \in E_{+}$, we construct a net as follows. Let $\mathscr{F}$ be the family of all finite sets of minimal ideals of $E$. $\mathscr{F} \times \mathbf{N}$, ordered by $(F, n) \geqq(G, m)$ if and only if $F \supset G$ and $n \geqq m$, is a directed set. If $(F, n) \in \mathscr{F} \times \mathbf{N}$, where $F=\{J(1), J(2), \cdots, J(k)\}$ let

$$
y_{(F, n)}=\left(I-P_{J(1)}\right) \cdots\left(I-P_{J(k)}\right) u+\sum_{i=1}^{k} P_{J(i)}(u) \wedge(j(i) / n)
$$

where $j(i) \in J(i)_{+},\|j(i)\|=1$. The net $y_{(F, n)}$ is downard directed and has infimum 0 . For if $0 \leqq z \leqq y_{(F, n)}$ for all $(F, n) \in \mathscr{F} \times \mathbf{N}$ then $0 \leqq P_{J} z \leqq P_{J} y_{(\{J\}, n)} \leqq$ $j / n$ for all minimal ideals $J$ and each $n \in \mathbf{N}$ (again $j \in J_{+},\|j\|=1$ ). Hence $P_{J} z=0$. As the linear span of the minimal ideals is order dense in $E, z=0$.

Now fix $(F, n)$. If $J$ is a minimal ideal, fix $j \in J_{+}$with $\|j\|=1$, and define $f_{J}$ by $P_{J}(x)=f_{J}(x) \cdot j$ for all $x \in E$. $f_{J}$ is a lattice homomorphism and lies in $E^{\prime}$. As $x_{\gamma} \rightarrow 0$ weakly we may find $\gamma_{0}$ such that $\left|f_{J}\left(x_{\gamma}\right)\right| \leqq n^{-1}$ for each $J \in F$ and all $\gamma \geqq \gamma_{0}$. Now $P_{J}\left(\left|x_{\gamma}\right| \wedge u\right)=\left|P_{J}\left(x_{\gamma}\right)\right| \wedge P_{J}(u) \leqq P_{J}(u) \wedge(j / n)$ if $J \in F$ and $\gamma \geqq \gamma_{0}$. It follows that $\left|x_{\gamma}\right| \wedge u \leqq y_{(F, n)}$ if $\gamma \geqq \gamma_{0}$ so that $\left(x_{\gamma}\right) u o$-converges to 0 .

In proving the equivalence of (i) and (iii) it would not have sufficed to consider only sequences in (i). Indeed if $m$ is any measure which is not purely atomic then $L^{\star}(m)$ provides a counterexample by virtue of: 
Proposition 2. Let $S$ be a compact quasi-Stonian space. Every sequence in $C(S)$ which converges weakly to 0 must uo-converge to 0 .

Proof. Let $f_{n} \rightarrow 0$ weakly, $f_{n} \in C(S)$. As $S$ is quasi-Stonian we may, for each $m \in \mathbf{N}$, form $\vee_{n=m}^{x}\left|f_{n}\right|$ in $C(S)$, (Schaefer (1974), Corollary of Proposition II.7.7, plus the fact that the $f_{n}$ 's must be norm, and hence order bounded in $C(S))$. Furthermore $N_{m}=\left\{s \in S: \vee_{n=m}^{\infty}\left|f_{n}(s)\right| \neq\left(\vee_{n=m}^{\infty}\left|f_{n}\right|\right)(s)\right\}$ is nowhere dense in $S$. It suffices to prove that $\Lambda_{m=1}^{\infty} \vee_{n=m}^{\infty}\left|f_{n}\right|=0$. If this fails we may find a non-empty open and closed $A \subset S$ and $\varepsilon>0$ for which $\vee_{n=m}^{\infty}\left|f_{n}\right| \geqq \varepsilon \chi_{A}$ for all $m$. As $A$ is of the second category $B=A \backslash\left(\bigcup_{m=1}^{\infty} N_{m}\right) \neq \varnothing$. If $s \in B$ then $\left(\vee_{n=m}^{\infty}\left|f_{n}\right|\right)(s)=\vee_{n=m}^{\infty}\left|f_{n}(s)\right|$ for all $m$. But $\left(\vee_{n=m}^{\infty}\left|f_{n}\right|\right)(s) \geqq \varepsilon$ for all $m$, whilst $\left|f_{n}(s)\right| \rightarrow 0$, as $f_{n} \rightarrow 0$ weakly. This contradiction establishes the claim.

It is of some interest that it does suffice to consider sequences if the Banach lattice $E$ has an order continous norm. For such an $E$ the condition that the linear span of the minimal ideals is order dense is equivalent to the norm closed convex hull of the extremal rays of $E_{+}$being the whole of $E_{+}$(an extremal ray of $E_{+}$is the positive part of a minimal ideal in $E$ ). Banach lattices with this property have been studied in Walsh (1968) and Wickstead (1975). Before proving the result we state a lemma.

LEMMA 3. Let $E$ be a Banach lattice with an order continuous norm, and for which $E_{+}$has no extremal rays. If $x \in E_{+}, f \in E_{+}^{\prime}$ and $f(x)>0$ there is $y \in E$ with $0 \leqq y \leqq x, y \wedge(x-y)=0$ and $\mathrm{f}(\mathrm{y})=\mathrm{f}(\mathrm{x}) / 2$.

Proof. As $E$ has an order continuous norm the order intervals in $E$ are weakly compact (Schaefer (1974), Theorem 11.5.10). The first two paragraphs of the part of the proof of Theorem 5 in Wickstead (1975) labelled "Main Proof" proves the result, using weak compactness of order intervals in place of norm compactness.

THEOREM 4. If $E$ is a Banach lattice with an order continuous norm then following are equivalent:

(i) Every net in E which converges weakly to 0 must uo-converge to 0 .

(ii) Every sequence in $E$ which converges weakly to 0 must uo-converge to 0 .

(iii) $E_{+}$is the norm closed convex hull of its extremal rays.

Proof. We have only to prove that (ii) implies (iii). The norm closed linear span of the extremal rays of $E_{+}$is an ideal in $E$, and hence, (Schaefer (1974), Theorem II.5.14) a band. As $E$ is Dedekind complete (Schaefer (1974), Theorem II.5.10) if (iii) fails the complementary band is non-zero. Thus to prove the result it suffices to suppose that $E \neq\{0\}$ and $E_{+}$has no 
extremal rays, and then to construct a sequence in $E$ which converges weakly to 0 , but does not uo-converge to 0 . Choose $f \in E_{+}^{\prime}\{\{0\}$, we may suppose (if necessary by replacing $E$ by the band complementary to $\{x \in E: f(|x|)=0\})$ that $f$ is strictly positive on $E . \mid\{0\}$.

Choose $y \in E_{+}$with $f(y)=1$. Using lemma 3 we may construct $z_{n, i} \in E_{+}$ $\left(n \in \mathbf{N}, 1 \leqq i \leqq 2^{n}\right)$ as follows:

$$
z_{1,1}+z_{1,2}=y, z_{1,1} \wedge z_{1,2}=0, \quad f\left(z_{1,1}\right)=f\left(z_{1,2}\right)=2^{-1} .
$$

If $z_{n, i}$ is defined then $z_{n+1,2 i-1}$ and $z_{n+1.2 i}$ are defined so that

$$
\begin{aligned}
& z_{n+1,2 i-1}+z_{n+1,2 i}=z_{n, i} \\
& z_{n+1,2 i-1} \wedge z_{n+1,2 i}=0 \\
& f\left(z_{n+1,2 i-1}\right)=f\left(z_{n+1,2 i}\right)=2^{-(n+1)} .
\end{aligned}
$$

Hence $\vee_{i=1}^{2 n} z_{n, i}=\sum_{i=1}^{2 n} z_{n, i}=y$ for all $n \in \mathbf{N}$. Define the sequence $\left(x_{m}\right)$ by $x_{2^{n+i}}=z_{n, i}$. The sequence $\left(x_{m}\right)$ does not uo-converge to 0 , for $w \geqq\left|x_{m}\right| \wedge y=$ $x_{m}$ for all $m \geqq m_{0}$. Choose $n \in \mathbf{N}$ with $2^{n} \geqq m_{0}$. Now $w \geqq x_{2^{n}+i}\left(1 \leqq i \leqq 2^{n}\right)$ so that $w \geqq y>0$.

It remains to prove that $\left(x_{m}\right)$ converges weakly to 0 . Let $X=$ $\left\{x_{m}: m \in \mathbf{N}\right\} \cup\{0\}$. I claim $X$ is weakly closed in the order interval $[0, y]$, and is hence weakly compact. Indeed, if $\left(s_{\gamma}\right)$ is a net in $X$ that converges weakly to $s \in[0, y]$ then either $s=0$ (in which case we have nothing to prove) or $f(s)>0$ (as $f$ is strictly positive on $E_{+} \mid\{0\}$ ). As $f\left(s_{\gamma}\right) \rightarrow f(s)$ and $f\left(s_{\gamma}\right)$ takes only the values $2^{-k}$, for $k \in \mathbf{N}, f\left(s_{\gamma}\right)$ must eventually be constant. Also there are, for each $k$, only finitely many distinct $s_{\gamma}$ with $f\left(s_{\gamma}\right)=2^{-k}$. Thus the net must eventually be constant, so $s \in X$.

As $f$ is strictly positive on $E_{+} \mid\{0\}, d(s, t)=f(|s-t|)$ defines a metric on $X$. The topology induced on $X$ by $d$ is coarser then the weak topology restricted to $X$. For if $\left(s_{\gamma}\right)$ is a net in $X$ converging weakly to $s$ then either $s=0$ or $\left(s_{\gamma}\right)$ is eventually constant (as we have just shown). In the latter case we have $d\left(s_{\gamma}, s\right)=0$ eventually, whilst in the former case $d\left(s_{\gamma}, 0\right)=$ $f\left(\left|s_{\gamma}-0\right|\right)=f\left(s_{\gamma}\right) \rightarrow 0$. It follows that the weak and $d$-topologies coincide on $X$. As $d\left(x_{2^{n}+i}, 0\right)=2^{-n}$ we see that $x_{m} \rightarrow 0$ for the $d$-topology and hence $x_{m} \rightarrow 0$ weakly.

\section{When does uo-convergence imply weak convergence?}

We deal with the reverse implication in this section. The answer to the problem posed in the title of this section is "only for finite dimensional spaces". Indeed such a space must satisfy the equivalent conditions of Theorem 5. If it were infinite dimensional we could choose disjoint $x_{n}$ with 
$\left\|x_{n}\right\|=1$. As in the proof of (i) $\Rightarrow$ (ii) in Theorem 5 we have $\left(n x_{n}\right)$ uoconverging to 0 , yet $\left\|n x_{n}\right\| \rightarrow \infty$ so it is impossible that $n x_{n} \rightarrow 0$ weakly. The situation is rather more interesting if we limit our attention to norm bounded nets.

THEOREM 5. For any Banach lattice $E$ the following are equivalent:

(i) Every norm bounded net in $E$ which uo-converges to 0 must converge weakly to 0 .

(ii) $E$ has an order continuous norm and every norm bounded disjoint sequence in $E$ converges weakly to 0 .

(iii) $E$ and $E^{\prime}$ have order continuous norms.

Proof. (i) $\Rightarrow$ (ii). (i) certainly implies that if $\left(x_{\gamma}\right)$ is a net decreasing to 0 then $x_{\gamma} \rightarrow 0$ weakly, and $x_{\gamma} \rightarrow 0$ in norm by (Schaefer (1974), Corollary to Theorem II.5.9). Thus $E$ has order continuous norm. If $\left(x_{n}\right)$ is a disjoint norm bounded sequence in $E$ then $\left(x_{n}\right) u o$-converges to 0 . Indeed if $u \in E_{+}$then for each $m \in \mathbf{N} \vee_{n=m}^{\infty}\left(\left|x_{n}\right| \wedge u\right)$ exists as $E$ is Dedekind complete (Schaefer (1974), Theorem II.5.10), and $\vee_{n=m}^{\infty}\left(\left|x_{n}\right| \wedge u\right) \searrow 0$ as the $x_{n}$ 's are disjoint. Thus (i) implies that $x_{n} \rightarrow 0$ weakly.

(ii) $\Rightarrow$ (i). Let $E$ have an order continuous norm and $\left(x_{\gamma}\right)$ be a norm bounded net in $E$ which uo-converges to 0 , but does not converge weakly to 0 . We construct a norm bounded disjoint sequence in $E$ which does not converge weakly to 0 . This will prove the implication. We note first that if $u \in E_{+}$then $\left(u \wedge\left|x_{\gamma}\right|\right)$ must, as $E$ has an order continuous norm, converge to 0 in norm.

If $\left(x_{\gamma}\right)$ does not converge weakly to 0 there is $f \in E_{+}^{\prime}$ such that $f\left(x_{\gamma}\right) \not 0$. By choosing a subnet of the $\left(x_{\gamma}\right)$ and an appropriate $f$ we may assume that $\left|f\left(x_{\gamma}\right)\right| \geqq 2$ for all $\gamma$. Thus we have $f\left(\left|x_{\gamma}\right|\right) \geqq 2$ for all $\gamma$.

We construct a bounded collection of $y_{n, i}$ in $E_{+}(n \in \mathbf{N}, 1 \leqq i \leqq n)$ with the properties that

$$
\begin{aligned}
& y_{n, i} \wedge y_{n, j}=0 \quad \text { if } \quad i \neq j \\
& f\left(y_{n, i}\right) \geqq 1+2^{-n} \\
& y_{n, i} \geqq y_{n+1, i} \quad \text { if } \quad n \in \mathbf{N}, \quad n \geqq i .
\end{aligned}
$$

The construction proceeds inductively. Choose any $\gamma_{1}$ and set $y_{1,1}=\left|x_{\gamma_{1}}\right|$. Now suppose we have defined $y_{n, 1}, \cdots, y_{n, n}$, we show how to choose $y_{n+1, i}(1 \leqq i \leqq$ $n+1)$.

Let $u=\vee_{i=1}^{n} y_{n, i}$. Choose $\gamma$ such that $f\left(u \wedge\left|x_{\gamma}\right|\right) \leqq 2^{-(n+1)}$. Set $y_{n+1, n+1}=$ $\left|x_{\gamma}\right|-u \wedge\left|x_{\gamma}\right|$, so that $f\left(y_{n+1, n+1}\right) \geqq 2-2^{-(n+1)} \geqq 1+2^{-(n+1)}$. Let $Q$ be the band projection onto the band generated by $y_{n+1, n+1}$. It is readily verified that $Q u \leqq u \wedge\left|x_{y}\right|$. We set $y_{n+1, i}=(I-Q) y_{n, i}(1 \leqq i \leqq n)$. We certainly have the 
disjointness condition and the monotonicity condition satisfied. Also $y_{n, i} \leqq u$ so $Q y_{n . i} \leqq Q u \leqq u \wedge\left|x_{\gamma}\right|(1 \leqq i \leqq n)$. Hence

$$
\begin{aligned}
f\left(y_{n+1, i}\right) & =f\left(y_{n, i}\right)-f\left(Q y_{n, i}\right) \\
& \geqq 1+2^{-n}-f\left(u \wedge\left|x_{\gamma}\right|\right) \\
& \geqq 1+2^{-n}-2^{-(n+1)} \\
& =1+2^{-(n+1)} .
\end{aligned}
$$

Finally note that each $y_{n, i}$ is dominated by some $\left|x_{y}\right|$, so the set of all of them is certainly norm bounded.

Let $z_{i}=\wedge_{n=i}^{\infty} y_{n, i}$. As the norm in $E$ is order continuous $f\left(z_{i}\right)=$ $\wedge_{n=i}^{\infty} f\left(y_{n, i}\right) \geqq 1$. Thus $\left(z_{i}\right)$ does not converge weakly to 0 . As the $z_{i}$ 's are clearly disjoint, we have the desired contradiction of (ii).

(ii) $\Rightarrow$ (iii). If $E$ has an order continuous norm but $E^{\prime}$ does not then $E$ contains a sublattice topologically order isomorphic with $l_{1}$ (Meyer-Nieberg (1973), Korollar 1.4). Let $e_{n}$ be the sequence in $l_{1}$ with $n$ 'th coordinate 1 and the remainder 0 , then $e_{n}$ does not converge weakly to 0 in $l_{1}$. Hence the corresponding sequence in $E$, which is disjoint, does not converge weakly to 0 yet is norm bounded, showing that (ii) fails.

(iii) $\Rightarrow$ (ii). If the sequence $\left(x_{n}\right)$ in $E$ is norm bounded, disjoint, and does not converge weakly to 0 , we may (as in the proof of (ii) $\Rightarrow$ (i)) suppose $x_{n} \geqq 0$ and that there is $f \in E_{+}^{\prime}$ with $f\left(x_{n}\right) \geqq 1$ for each $n$. It is routine to verify that the norm on the linear span of the $x_{n}$ 's is equivalent to the norm $\left\|\sum \alpha_{i} x_{i}\right\|=$ $\sum\left|\alpha_{i}\right|$. Thus the closed linear span of the $x_{n}$ 's is isomorphic to $l_{1}$, and (iii) fails by Meyer-Nieberg (1973), Korollar 1.4.

It does not suffice to consider only sequences in (i), as is shown by the space of all continous real valued functions on the one point compactification of an uncountable discrete space. A norm bounded uo-convergent sequence there must converge in norm, so certainly weakly (see Luxemburg and Zaanen (1964), Example 33.1).

We note that if $E_{+}$is the norm closed convex hull of its extremal rays then the linear span of the extremal rays of $E_{+}^{\prime}$ is order dense in $E_{+}^{\prime}$. Thus if $E_{+}^{\prime}$ also has an order continuous norm then the norm closed convex hull of the extremal rays in $E_{+}^{\prime}$ is the whole of $E_{+}^{\prime}$. We may now combine Theorems 1 and 5 to obtain:

THEOREM 6. For any Banach lattice $E$ the following are equivalent:

(i) For norm bounded nets in $E$ weak and uo-convergence coincide.

(ii) The positive cones in both $E$ and $E^{\prime}$ are the norm closed convex hull of their extremal rays. 
Any Banach lattice satisfying the conditions of Theorem 6 will certainly satisfy those of Theorem 5. This may be seen directly by arguing that (ii) of Theorem 5 is equivalent to order intervals in $E$ and $E^{\prime}$ being norm (and hence weakly) compact by Theorem 5 of Wickstead (1975), and this implies that $E$ and $E^{\prime}$ have order continous norms by Schaefer (1974), Theorem II.5.10.

\section{References}

R. DeMarr (1964), 'Partially ordered linear spaces and locally convex linear topological spaces', Illinois J. Math. 8, 601-606.

G. Köthe (1969), Topological Vector Spaces I (Springer-Verlag, 1969).

W. A. J. Luxemburg and A. C. Zaanen (1964), 'Notes on Banach function spaces X', Indag. Math. 26, 493-506.

P. Meyer-Nieberg (1973), 'Zur schwachen Kompaktheit in Banachverbänden', Math. Z. 134, 303-315.

H. H. Schaefer (1974), Banach Lattices and Positive Operators (Springer-Verlag, 1974).

B. Walsh (1968), 'On characterising Köthe sequence spaces as vector lattices', Math. Ann. 175 , 253-256.

A. W. Wickstead (1975), 'Compact subsets of partially ordered Banach spaces', Math. Ann. 212, $271-284$

A. Wirth (1975), 'Relatively uniform Banach lattices', Proc. Amer. Math. Soc. 52, 178-180.

Department of Pure Mathematics, The Queen's University of Belfast. 